\title{
Hyperbaric oxygen therapy cognitive function in a rat model of mild cognitive impairment via ERK signaling
}

\author{
Yuerong Lin ${ }^{1,2 \#}$, Xianzhong Lin ${ }^{1,2 \#}$, Xiaohong Zheng ${ }^{1,2}$, Fang Liu ${ }^{3}$, Chen Ye $^{1,2}$, Linshen Huang ${ }^{1,2}$, \\ Qian Zhou ${ }^{1,2}$, Ting Chen ${ }^{1,2}$, Lanying Lin ${ }^{1,2}$ \\ ${ }^{1}$ Department of Anesthesiology, the First Affiliated Hospital of Fujian Medical University, Fuzhou, China; ${ }^{2}$ Institute of Anesthesiology, Fujian \\ Medical University, Fuzhou, China; ${ }^{3}$ Department of Hyperbaric Oxygen Therapy, the First Affiliated Hospital of Fujian Medical University, Fuzhou, \\ China \\ Contributions: (I) Conception and design: Y Lin, X Lin, L Lin; (II) Administrative support: L Lin; (III) Provision of study materials or patients: C Ye, \\ F Liu; (IV) Collection and assembly of data: X Zheng, Q Zhou; (V) Data analysis and interpretation: Y Lin, L Huang; (VI) Manuscript writing: All \\ authors; (VII) Final approval of manuscript: All authors. \\ "These authors contributed equally to this work. \\ Correspondence to: Lanying Lin; Xianzhong Lin. Department of Anesthesiology, the First Affiliated Hospital of Fujian Medical University, 20 \\ Chazhong Road, Fuzhou, China. Email: linlanying@163.com; lxzyjs@fjmu.edu.cn.
}

Background: The development of novel treatment strategies to reverse or impede cognitive dysfunction associated with mild cognitive impairment (MCI) has gained attention in recent times. Meanwhile, hyperbaric oxygen (HBO) has been widely used as a neuroprotective therapy that can promote recovery of damaged neurons. The aim of this study was to investigate the effects of $\mathrm{HBO}$ on cognitive function in rats with MCI and to clarify the associated mechanisms.

Methods: We established a D-galactose-induced MCI rat model and evaluated the role of extracellularregulated kinase (ERK) signaling in HBO therapy for cognitive function using a specific inhibitor, U0126. All Rats were randomly assigned to four groups with 12 rats per group: normal control group; D-gal model group (group MCI); D-gal + HBO group (group HBO); D-gal + HBO + U0126 group (group U0126). We evaluated cognitive function by Morris water maze and pathological changes by hematoxylin and eosin (HE) staining of hippocampal slices. Enzyme-linked immunosorbent assay (ELISA) was used to detect the levels of caspase 3, while total ERK1/2 and phospho-ERK1/2 were assessed by Western blotting.

Results: Shorter escape latency was observed in the HBO group as compared to the MCI group, which was to some extent reversed by U0126. Similarly, the HBO group showed the highest number of platform crossings as compared to the MCI and U0126 groups. Pathological analysis also showed less apoptosis and better hippocampal cell morphology in the HBO group. Caspase 3 levels also differed significantly, with lowest expression in the HBO group as compared to the MCI and U0126 groups. The levels of total ERK1/2 and p-ERK1/2 were more elevated in the HBO group as compared to the MCI group.

Conclusions: This study found that HBO treatment has a protective effect on early cognitive dysfunction in rats with MCI. HBO therapy may act through ERK signaling, which inhibits apoptosis and protects cognitive function.

Keywords: Hyperbaric oxygen (HBO); extracellular regulated protein kinase; mild cognitive impairment (MCI); neuroprotection

Submitted Aug 10, 2020. Accepted for publication Sep 11, 2020.

doi: 10.21037/apm-20-1716

View this article at: http://dx.doi.org/10.21037/apm-20-1716 


\section{Introduction}

Cognitive dysfunction can manifest as deficits in orientation, memory, attention, numeracy, or language ability, and may result in daily or social impairment, such as aphasia, loss of recognition, or change in mental or neurological activity. Mild cognitive impairment (MCI), a type of cognitive dysfunction, is a clinical condition between cognitive decline and normal aging. Around 16\% of MCI cases progress to dementia each year, with this trend increasing year by year (1). Epidemiological studies have shown that an MCI case may result in one of four outcomes: stable condition, progress to dementia, recovery to normal cognition through intervention, or death. Thus, as MCI seems to respond to intervention, diagnosis and treatment of MCI have attracted the attention of many researchers.

There are various treatments which are no uniform standard for MCI. Currently, the Food and Drug Administration (FDA) has no approved drugs for the treatment of MCI. Drug treatments usually are based on Alzheimer's disease, such as cholinesterase inhibitors (2). Studies have also shown that repeated transcranial magnetic stimulation can significantly change cortical excitability, improve cognitive function, and treat MCI (3).

Hyperbaric oxygen ( $\mathrm{HBO}$ ) has been widely used as a classic neuroprotective therapy that can promote recovery of damaged neurons $(4,5)$. This treatment is non-invasive, safe, and its clinical application is highly controllable. The main mechanisms involved in HBO therapy are increased blood oxygen content, inhibition of hippocampal neuronal apoptosis, and reduction in inflammation (6-8).

Mitogen-activated protein kinase (MAPK) is a type of protein kinase that regulates various physiological processes by phosphorylating the corresponding transcription factors, cytoskeletal proteins, and various protein substrates. It is involved in cell proliferation, differentiation, survival, and death. The extracellularregulated kinase (ERK) signaling pathway is a classical MAPK signal transduction pathway. Activated ERK1/2 can enter the nucleus and phosphorylate downstream substrates, which further activate other related transcription factors to produce specific biological effects. Some research results have indicated that ERK signaling pathway plays a protective role in brain injury, can inhibit neuronal apoptosis, and improve its cognitive function $(9,10)$. U0126 is a highly effective and selective inhibitor of MAPK kinases MEK1 and MEK2. It can block the phosphorylation of ERK by inhibiting the activity of MEK1/2, thereby blocking the ERK1/2 signaling pathway and inhibiting the MAPK cascade.

Based on the above studies, we aimed to investigate the effects and possible mechanisms of $\mathrm{HBO}$ administration on cognitive function in a rat model MCI. We present the following article in accordance with the ARRIVE reporting checklist (available at http://dx.doi.org/10.21037/apm-201716).

\section{Methods}

\section{Drugs and reagents}

D-gal was procured from AMRESCO Inc. (OH, USA). U0126-EtOH was obtained from MedChemExpress LLC (NJ, USA). Caspase 3 ELISA kit was purchased from Nanjing Jiancheng Bioengineering Institute (Nanjing, China). Rabbit polyclonal anti-ERK1/2 and antiphospho-ERK1/2 antibodies were supplied by Hangzhou HuaAn Biotechnology Co. Ltd. (Hangzhou, China). Horseradish peroxidase (HRP)-labeled goat anti-rabbit immunoglobulin $\mathrm{G}$ ( $\mathrm{IgG}$ ) was provided by Abcam PLC (Cambridge, UK).

\section{Animals}

The study was approved by the animal care committee of the First Affiliated Hospital of Fujian Medical University(Fuzhou, China). All experiments were conducted in accordance with EU Directive 2010/63/EU for animal experiments. Sprague Dawley (SD) rats (male, 3-month-old, 200 to 300 g, Minhou Wu Experimental Animal Co. Ltd., China) were housed in a temperature-controlled room (22$26^{\circ} \mathrm{C}$ ) with a $12 \mathrm{~h}$ light-dark cycle and food and drinking water provided ad libitum. Rats that were visually impaired or could not swim were excluded from the study. Rats were acclimatized to the environment for 1 week before the experiments.

Rats were randomly assigned to four groups with 12 rats per group: normal control group, without any intervention; D-gal model group (group MCI), subcutaneous injection of $1,000 \mathrm{mg} \cdot \mathrm{kg}^{-1} \cdot \mathrm{d}^{-1} \mathrm{D}$-gal (in physiological saline, $100 \mathrm{mg} / \mathrm{mL}$ ) for 1 week; D-gal + HBO group (group HBO), D-gal injections followed by HBO treatment; D-gal + HBO + U0126 group (group U0126), D-gal injection followed by $\mathrm{HBO}$ treatment along with daily intraperitoneal injection of U0126 (5 mg: $\left.\mathrm{kg}^{-1} \cdot \mathrm{d}^{-1}\right)$ before HBO treatment. 


\section{HBO administration}

Rats in the HBO group and U0126 group were placed in a $\mathrm{HBO}$ chamber, flushed with pure oxygen to achieve an oxygen concentration of $95 \%$ or more. Next, the cabin was gradually pressurized to $2.5 \mathrm{~atm}$ at a rate of $0.2 \mathrm{~atm} \cdot \mathrm{min}^{-1}$ for $60 \mathrm{~min}$. Thereafter, the pressure was dropped to normal at the same speed. This treatment was administered once a day for 5 days (11).

\section{U0126 injection}

U0126 is a highly selective inhibitor of MEK1/2. As per the manufacturer's instructions, $5 \mathrm{mg}$ of U0126 was dissolved in a mixture of $0.655 \mathrm{~mL}$ of dimethyl sulfoxide (DMSO) and $0.655 \mathrm{~mL}$ of $1 \mathrm{M}$ of phosphate-buffered saline (PBS) and diluted in $12 \mathrm{~mL}$ of physiological saline. Rats of group U0126 were intraperitoneally injected with $5 \mathrm{mg} \cdot \mathrm{kg}^{-1} \cdot \mathrm{d}^{-1}$ before HBO treatment (12).

\section{Behavioral analysis}

The Morris water maze test is an important research tool for the assessment of learning and memory function in rodents. The water maze consisted of a circular pool with a diameter of $180 \mathrm{~cm}$ and a depth of $80 \mathrm{~cm}$. An escape platform, a $12 \mathrm{~cm}$ diameter transparent disc, was located $2 \mathrm{~cm}$ below the water surface and was placed in the IV quadrant in this experiment. The pool water was made opaque black by edible bamboo charcoal powder so that the swimming path could be accurately tracked by the Supermaze software.

The Morris water maze test includes two phases: navigation training and a spatial probe test. During navigation training, a quadrant was randomly selected as the starting point, and the rats were individually placed in the pool, facing the pool wall. Time taken to reach the platform or escape latency was recorded for each rat. If a rat was unable to locate the platform within $90 \mathrm{~s}$, it was guided to the platform and was made to stay on the platform for $15 \mathrm{~s}$; escape latency of these rats was recorded as $90 \mathrm{~s}$. Each rat was trained from all four quadrants in sequence for 5 consecutive days. Throughout the experiment, light intensity and the surrounding environment were kept unchanged. The training was followed by spatial probe test, where the escape platform was removed from the pool and each rat was placed in the quadrant opposite to the original platform quadrant. The number of crossings of the platform within $60 \mathrm{~s}$ was recorded for each rat.

\section{Analysis of caspase 3, $t$-ERK1/2, and p-ERK1/2}

The hippocampal tissues of all groups (8 rats in each group) were taken out, and were then ground after rapid cooling with liquid nitrogen. The ground tissues were fully split and centrifuged so the supernatant could be collected. The protein concentration was determined by bicinchoninic acid (BCA) method. Then, the expression of t-Erk1/2 and p-ErK 1/2 was detected by Western blotting (t-Erk1/2 Antibody, Rabbit\#et1601-29. HA, Nanjing, China; p-ErK 1/2 Antibody, Rabbit\# rt1206, HA, Nanjing, China). Grayscale analysis was performed by Image J. The other half of the supernatants were used to measure the concentrations of caspase 3 by ELISA, through use of a corresponding ELISA kit (Caspase-3 ELISA kit, Rabbit\#er30804, HA, Nanjing, China). According to the manufacturer's instructions, the absorbance was detected at $450 \mathrm{~nm}$, and a standard curve was delineated based on the absorbance of standards. The loading quantity of each hole was $100 \mu \mathrm{L}$.

\section{Pathological evaluation}

All rats (4 rats in each group) were anesthetized by an intraperitoneal injection of $0.3 \%$ pentobarbital sodium $\left(50 \mathrm{mg} \cdot \mathrm{kg}^{-1}\right)$ after $12 \mathrm{~h}$ fasting. The heart and liver were exposed, and an incision was made in the pericardium. A needle was vertically inserted into the apex and fixed. Next, the right atrial appendage was cut with small scissors, and saline perfusion was started at a flow rate of about $50 \mathrm{~mL} \cdot \mathrm{min}^{-1}$. Upon a change in the color of the liver to white, $4 \%$ paraformaldehyde fixative was infused. The brain was carefully taken out and put into the fixative for another $24 \mathrm{~h}$. The fixed brain was paraffin-embedded, and the hippocampus was serially sectioned and stained with hematoxylin and eosin (HE). Pathological changes in the hippocampus were observed under $10 x$ and $40 \times$ light microscopes.

\section{Statistical analysis}

Data are expressed as mean \pm standard deviation $(\bar{x} \pm s)$ under the normal distribution. One-way analysis of variance (ANOVA) was used to compare body weights, swimming speed of rats before and after modeling, number of platform crossings, caspase 3, t-ERK1/2, and p-ERK1/2 levels. When the variances were equal, comparison between groups 
Table 1 Rats weights before and after modeling $(\mathrm{n}=12)$

\begin{tabular}{lccccc}
\hline Group & Weight before modeling $(\mathrm{g})$ & $\mathrm{F}$ & $\mathrm{P}$ & Weight after modeling $(\mathrm{g})$ & $\mathrm{F}$ \\
\hline Sham & $313.58 \pm 10.33$ & 0.701 & 0.556 & $331.83 \pm 6.97$ & 2.144 \\
$\mathrm{MCl}$ & $312.75 \pm 8.52$ & & & $328.33 \pm 5.91$ \\
HBO & $318.08 \pm 10.20$ & & $334.75 \pm 3.98$ \\
U0126 & $314.58 \pm 9.64$ & & & $332.58 \pm 7.72$ \\
\hline
\end{tabular}

Table 2 Rats swimming speed before and after modeling $(\mathrm{n}=12)$

\begin{tabular}{lccccc}
\hline Group & Speed before modeling $(\mathrm{mm} / \mathrm{s})$ & $F$ & $P$ & Speed after modeling $(\mathrm{mm} / \mathrm{s})$ & $\mathrm{F}$ \\
\hline Sham & $231.63 \pm 18.39$ & 0.473 & 0.702 & $240.18 \pm 23.97$ & $238.53 \pm 17.01$ \\
$\mathrm{MCl}$ & $236.26 \pm 8.86$ & & & $233.44 \pm 18.07$ \\
$\mathrm{HBO}$ & $232.44 \pm 20.10$ & & & $225.19 \pm 18.32$ \\
U0126 & $228.74 \pm 12.38$ & & & 247 \\
\hline
\end{tabular}
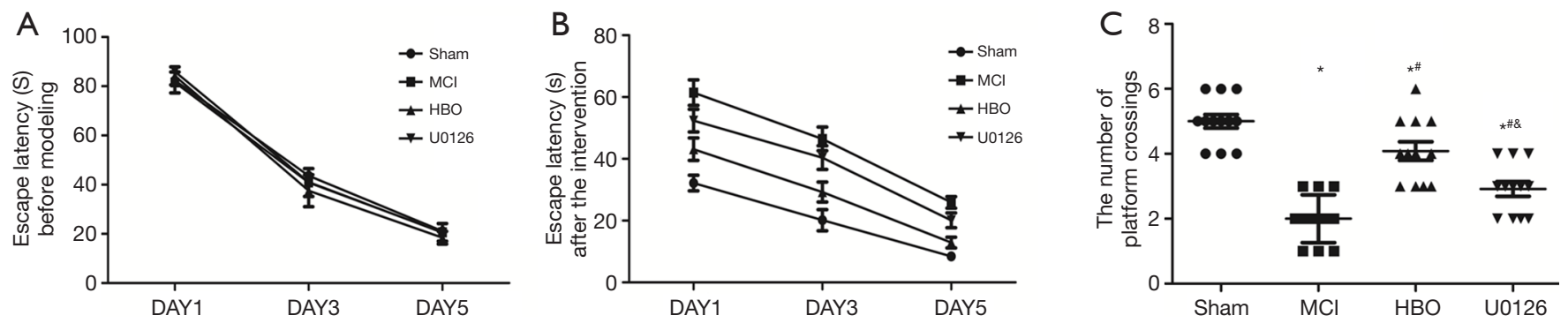

Figure 1 Results of the Morris water maze test. (A) The escape latency before modeling. There was no significant difference among all groups; (B) the escape latency after the intervention. Shorter escape latencies were noted in the HBO group and U0126 group than in the MCI group. The HBO group showed a shorter escape latency than the U0126 group; (C) the number of platform crossings. *, $\mathrm{P}<0.05$ vs. normal control group; ", $\mathrm{P}<0.05$ vs. MCI group; ${ }^{*}, \mathrm{P}<0.05$ vs. HBO group.

was performed by one-way ANOVA, and lease significant difference (LSD) $t$-test was used for multiple comparisons; when the variances were not uniform, one-way ANOVA (Welch correction) and Dunnett T3 were used. Escape latency was compared using repeated-measures ANOVA. A $\mathrm{P}$ value $<0.05$ was considered as statistically significant. All data were analyzed by SPSS25.0 statistical software.

\section{Results}

\section{General condition}

All rats included in the study showed good mental state and normal behavior in daily life. No difference was observed in body weights and swimming speed before and after modeling $(\mathrm{P}>0.05)$ (Tables 1,2).

\section{Bebavioral analysis}

In the Morris water maze test, no significant difference was observed in escape latency before modeling (Figure 1A). Moreover, the escape latency gradually decreased with training. However, the escape latency differed significantly in all groups after the intervention $(\mathrm{P}<0.001)$ (Figure $1 B)$. Shorter escape latencies were noted in the HBO group $(\mathrm{P}<0.001)$ and the $\mathrm{U} 0126$ group $(\mathrm{P}<0.001)$ than in the MCI group. Furthermore, the HBO group showed a shorter escape latency than the $\mathrm{U} 0126$ group $(\mathrm{P}<0.001)$.

The number of platform crossings also showed a significant difference between the four groups $(\mathrm{P}<0.001)$ 

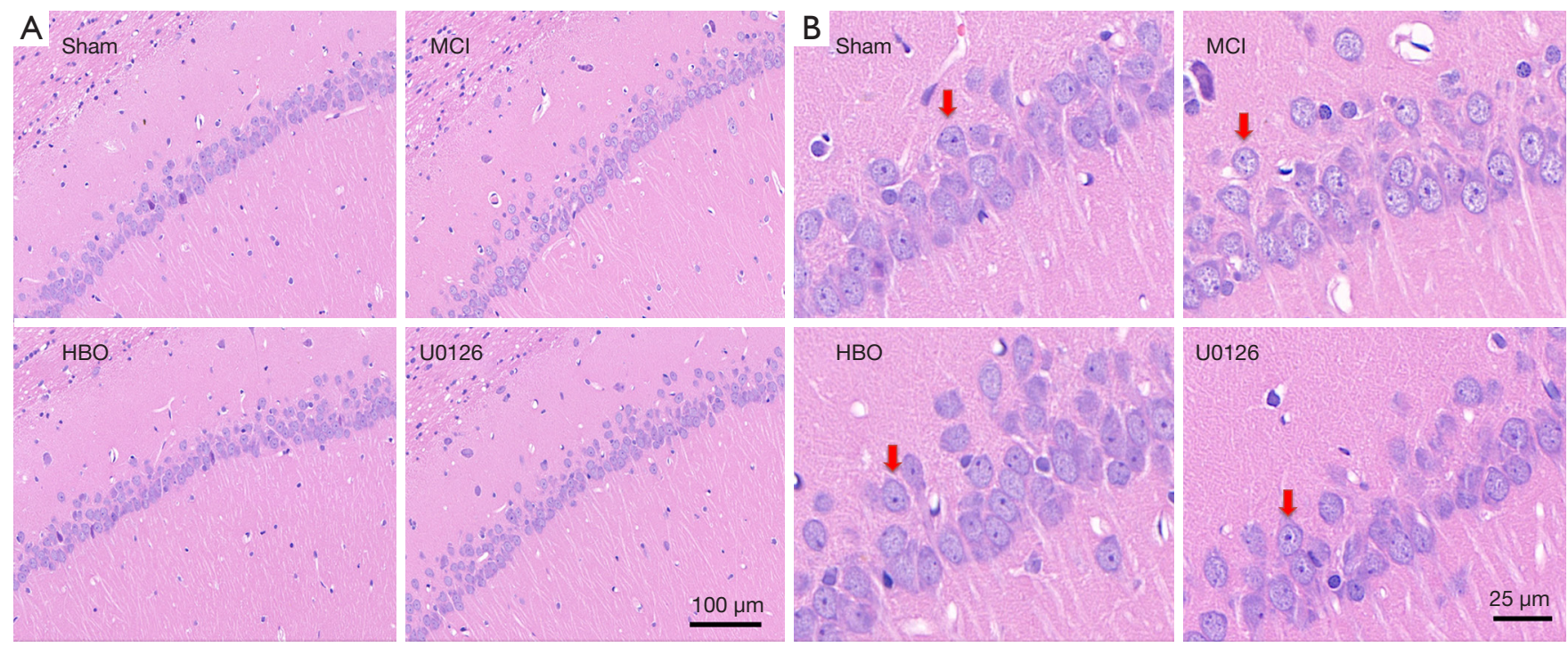

Figure 2 Pyramidal cells in the CA1 region of the hippocampus by hematoxylin and eosin staining at $10 \times(\mathrm{A})$ and $40 \times(\mathrm{B})$. Bar $=100 \mu \mathrm{m}(\mathrm{A})$ and $25 \mu \mathrm{m}$ (B). In the control group, the cells exhibited normal density and structure, with lightly stained, large, and round nuclei. The MCI group showed obvious apoptosis of the pyramidal cells, disordered cell morphology, and significantly decreased cell number. Less apoptosis and better hippocampal cell morphology were apparent in the HBO group. In the U0126 group, the degree of change was between that of the MCI and the HBO groups.

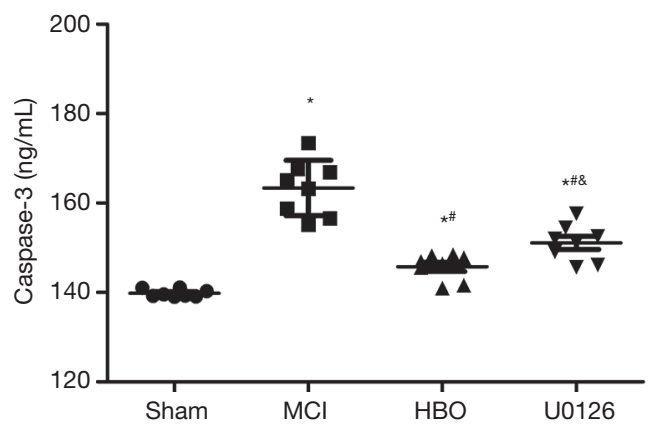

Figure 3 The level of caspase 3 in hippocampal tissues of rats. The graph shows that there was a significant difference among the four groups in relation to the content estimated by caspase 3. Data are presented as mean $\pm \mathrm{SD}$ ( $\mathrm{n}=8$ per group). *, $\mathrm{P}<0.05$ vs. normal control group;, $\mathrm{P}<0.05$ vs. MCI group; ${ }^{\&}, \mathrm{P}<0.05$ vs. HBO group.

(Figure 1C). Compared with the MCI group, the number of platform crossings increased significantly in the $\mathrm{HBO}$ $(\mathrm{P}<0.001)$ and $\mathrm{U} 0126$ groups $(\mathrm{P}=0.009)$, with maximum crossings by the $\mathrm{HBO}$ group $(\mathrm{P}=0.001)$.

\section{HE staining}

Pathological examination of brain sections by HE staining showed that pyramidal cells in the CA1 region of the hippocampus were arranged closely in the control group. The cells exhibited normal density and structure, with lightly stained, large, and round nuclei. MCI group showed obvious apoptosis of the pyramidal cells, disordered cell morphology, and significantly decreased cell number. More cells were condensed and dark-stained. In the HBO group, the number and density of pyramidal cells were almost normal, and only minimal cell apoptosis was noted, while in the U0126 group, the degree of cell damage was in between the MCI and the HBO groups (Figure 2).

\section{Caspase 3, t-ERK1/2, and p-ERK1/2 levels}

A significant difference was observed in caspase 3 levels in the four groups $(\mathrm{P}<0.001)$ (Figure 3). Compared with the control group, the MCI group $(\mathrm{P}<0.001), \mathrm{HBO}$ group $(\mathrm{P}=0.006)$, and $\mathrm{U} 0126$ group $(\mathrm{P}<0.001)$ showed significantly elevated caspase 3 levels. However, the HBO group demonstrated the lowest caspase 3 levels among these three groups.

The levels of $\mathrm{t}-\mathrm{ERK} 1 / 2(\mathrm{P}<0.001)$ and $\mathrm{p}-\mathrm{ERK} 1 / 2$ $(\mathrm{P}<0.001)$ (Figure 4) were also found to be significantly altered in all the groups. Compared with the control group, t-ERK1/2 and p-ERK1/2 were elevated in the MCI group 
A
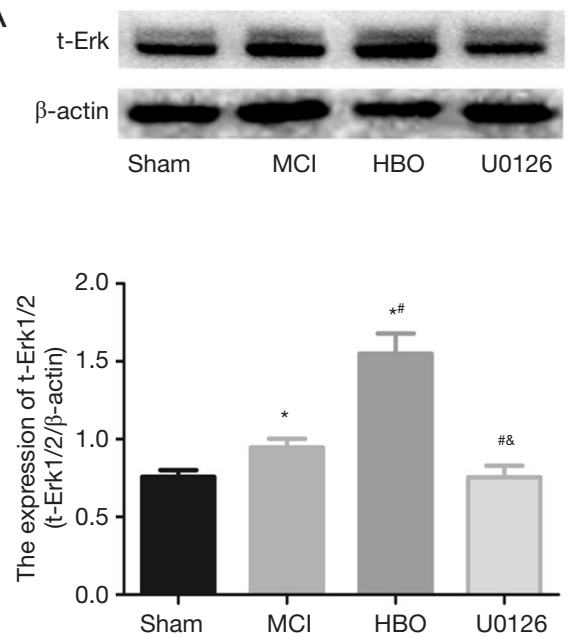

B
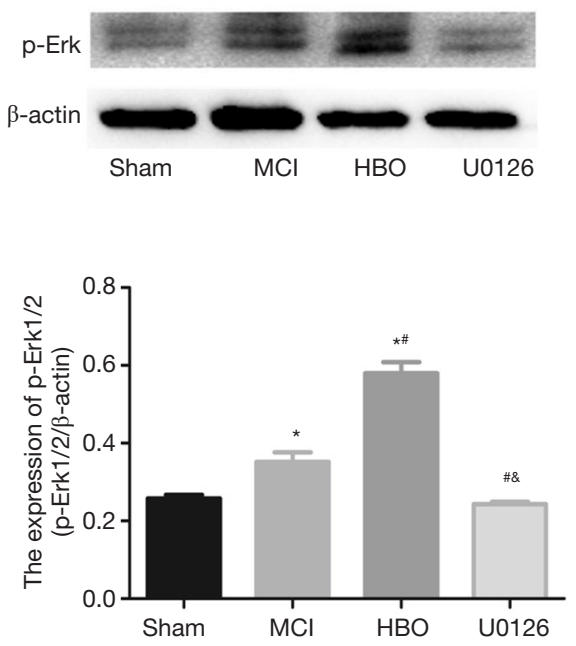

Figure 4 The levels of $\mathrm{t}$-Erk1/2 (A) and p-Erk1/2 (B) in the hippocampal tissues of rats. The expression of $\mathrm{t}$-Erk1/2 and p-ErK1/2 was detected by Western blotting. Data are presented as mean $\pm \mathrm{SD}$ ( $\mathrm{n}=8$ per group). ${ }^{*}, \mathrm{P}<0.05$ vs. normal control group; ${ }^{*}, \mathrm{P}<0.05$ vs. MCI group; ${ }^{\mathrm{*}}, \mathrm{P}<0.05$ vs. HBO group.

$(\mathrm{P}=0.025, \mathrm{P}<0.001)$ and $\mathrm{HBO}$ group $(\mathrm{P}<0.001, \mathrm{P}<0.001)$, and were the highest in the HBO group. No significant change in t-ERK1/2 $(\mathrm{P}=0.98)$ and $\mathrm{p}-\mathrm{ERK} 1 / 2(\mathrm{P}=0.40)$ level was observed between the U0126 group and the control group.

\section{Discussion}

Mild cognitive impairment has attracted attention in recent times. This condition can either gradually progress to dementia or remain stable without further deterioration. In some cases, it can be reversed to a near-normal cognitive state. Therefore, there is a need for identification and testing of potential therapeutic strategies that can help impede or reverse MCI progression. HBO therapy, a noninvasive treatment commonly used in clinics, has been shown to improve neurological function by inhibiting neuroinflammation and reducing cerebral edema and apoptosis, thereby ameliorating cognitive function. An ERK1/2 signaling pathway is a common pathway associated with multiple cellular processes; it regulates cell proliferation, apoptosis, and survival. Hsieh et al. reported that the proliferative effects of hyperbaric $\mathrm{O}_{2}$ on osteoblasts may contribute to the recruitment of osteoblasts at the fracture site by ERK signaling (13). HBO also might promote neural stem cell proliferation by activating ERK signaling and was shown to play an important role in neuroprotection after traumatic brain injury (14). These studies indicate that $\mathrm{HBO}$ is closely related to ERK signaling and cognitive function. Therefore, the present study was designed to investigate the effects of $\mathrm{HBO}$ on the ERK signaling pathway and cognitive function in rats with MCI.

In this study, an MCI rat model was established by subcutaneous injections of D-gal $\left(1,000 \mathrm{mg} \cdot \mathrm{kg}^{-1} \cdot \mathrm{d}^{-1}\right)$ for 1 week, which is known to induce aging. The success rate and stability of this model were confirmed in our previous study (15). This method results in impaired cognitive function, especially in memory, without causing any behavioral abnormalities.

The mechanism behind this model is that a high content of D-gal influences glucose metabolism, produces excessive oxygen-free radicals, and disrupts antioxidant defense systems, which eventually results in aging (16). Studies have also shown that D-gal can accelerate caspasemediated neuronal apoptosis and inhibit neurogenesis (17). Furthermore, to the best of our knowledge, this is the first study that has used this model to test HBO therapy and the role of ERK signaling pathway in MCI. The results of our study are consistent with previous studies that have demonstrated HBO therapy as an effective strategy in MCI rats (18).

Previous investigations on different animal models suggest that HBO can improve cognitive function (19). However, its correlation with ERK signaling pathway and cognitive function remains unexplored. We used the 
Morris water maze to test the cognitive function in different groups. Our study demonstrated that the cognitive function of the HBO group was better compared to that of the MCI group, and improvement in the cognitive function of the U0126 group was lower than that of the HBO group. Studies on the ERK signaling pathway have shown its contrasting roles in apoptosis stress, and other stimuliinduced activation of ERK signaling pathway has both been shown to cause $(20)$ and inhibit $(8,21,22)$ apoptosis. Caspase 3 is a well-established and sensitive marker of apoptosis. In our study, we assessed caspase 3 levels in brain homogenates and showed that the degree of apoptosis decreases after HBO treatment. However, upon U0126 treatment, which is known to inhibit ERK1/2 phosphorylation, caspase 3 levels were found to be increased. These results suggest that antiapoptotic effects are diminished after U0126 administration. Furthermore, we conducted a pathological analysis of the hippocampal sections and showed that the degree of apoptosis in the CA1 region of the MCI group was graver than that of the HBO group. The hippocampal apoptosis in the U0126 group was in between those of the other two groups. We further measured the levels of t-ERK1/2 and p-ERK1/2 in the brain samples and found that the levels were significantly higher in the HBO group, while a lower expression was observed in the U0126 group. Therefore, we believe that HBO may play a neuroprotective role and improve cognitive function by activating the ERK signaling pathway.

Something specific about the outcomes in relation to the model used. ERK signaling pathway plays a pivotal role in multiple physiological processes and may exert different effects on different animal models. Secondly, ERK may activate different downstream pathways at different times. Therefore, the time-point of stimulation and treatment might also have affected our results. Studies have shown that the ERK signaling pathway plays a dual role in hypoxic brain injury. Early high ERK expression has a protective effect on hypoxic neurons; however, long-term activation of ERK accelerates neuronal apoptosis (23).

A few limitations in our study should also be addressed. Firstly, the D-gal used in this study to establish a rat model of mild cognitive dysfunction alters cell signaling pathways and physiology. This study only explored the mechanism of $\mathrm{HBO}$ from the perspective of apoptosis and the ERK signaling pathway. Moreover, the time-point of HBO therapy in this study was selected after successful establishment of the rat model, and comparison of HBO therapy at different time-points was not investigated. Lastly, in this study, the ERK signaling pathway was evaluated only once, and a shift in ERK expression was not described, which may not accurately reflect the mechanism of the ERK signaling pathway.

Based on the above results, we believe that $\mathrm{HBO}$ administration may confer a therapeutic effect on early cognitive dysfunction in rats MCI. This treatment strategy may act through the ERK signaling pathway, which is known to inhibit apoptosis and ameliorate cognitive function.

\section{Acknowledgments}

Funding: This work was supported by the Natural Science Foundation of Fujian Province of China (2018J01178).

\section{Footnote}

Reporting Checklist: The authors have completed the ARRIVE reporting checklist. Available at http://dx.doi. org/10.21037/apm-20-1716

Data Sharing Statement: Available at http://dx.doi. org/10.21037/apm-20-1716

Conflicts of Interest: All authors have completed the ICMJE uniform disclosure form (available at http://dx.doi. org/10.21037/apm-20-1716). The authors have no conflicts of interest to declare.

Ethical Statement: The authors are accountable for all aspects of the work in ensuring that questions related to the accuracy or integrity of any part of the work are appropriately investigated and resolved. The study was approved by the animal care committee of the First Affiliated Hospital of Fujian Medical University (Fuzhou, China). All experiments were conducted in accordance with EU Directive 2010/63/EU for animal experiments.

Open Access Statement: This is an Open Access article distributed in accordance with the Creative Commons Attribution-NonCommercial-NoDerivs 4.0 International License (CC BY-NC-ND 4.0), which permits the noncommercial replication and distribution of the article with the strict proviso that no changes or edits are made and the original work is properly cited (including links to both the formal publication through the relevant DOI and the license). See: https://creativecommons.org/licenses/by-nc-nd/4.0/. 


\section{References}

1. Qiu C, Johansson G, Zhu F, Kivipelto M, Winblad B. Prevention of cognitive decline in old age-varying effects of interventions in different populations. Ann Transl Med 2019;7:S142.

2. Edmonds EC, MArd MC, Edland SD, et al. Unmasking the benefits of donepezil via psychometrically precise identification of mild cognitive impairment: a secondary analysis of the ADCS vitamin $\mathrm{E}$ and donepezil in MCI study. Alzheimers Dement 2018;4:11-8.

3. Drumond Marra HL, Myczkowski ML, Maia Memória C, et al. Transcranial Magnetic Stimulation to Address Mild Cognitive Impairment in the Elderly: A Randomized Controlled Study. Behav Neurol 2015;2015:287843.

4. Chung E, Zelinski LM, Ohgami Y, et al. Hyperbaric oxygen treatment induces a 2-phase antinociceptive response of unusually long duration in mice. J Pain 2010;11:847-53.

5. Zhao BS, Meng LX, Ding YY, et al. Hyperbaric oxygen treatment produces an antinociceptive response phase and inhibits astrocyte activation and inflammatory response in a rat model of neuropathic pain. J Mol Neurosci 2014;53:251-61.

6. Thom SR. Hyperbaric oxygen: its mechanisms and efficacy. Plast Reconstr Surg 2011;127:131S-41S.

7. Zhang XG, Wang GH, Li YC, et al. The influences of hyperbaric oxygen on the oxidative stress variables and pro-/anti-inflammatory cytokines in rats after traumatic brain injury. Zhongguo Ying Yong Sheng Li Xue Za Zhi 2010;26:373-5.

8. Gossett WA, Rockswold GL, Rockswold SB, et al. The safe treatment, monitoring and management of severe traumatic brain injury patients in a monoplace chamber. Undersea Hyperb Med 2010;37:35-48.

9. He J, Xue R, Wu G. Role of extracellular signal-regulated kinase in neuronal apoptosis of hippocampns induced by global cerebral ischemia-reperfusion in rats. Chinese Journal of Anesthesiology 2008;28:459-62 .

10. Li F, Omori N, Sato K, et al. Coordinate expression of survival p-ERK and proapoptotic cytochrome c signals in rat brain neurons after transient MCAO. Brain Res 2002;958:83-8.

11. Xiong L, Zhu Z, Dong H, et al. Hyperbaric oxygen preconditioning induces neuroprotection against ischemia in transient not permanent middle cerebral artery occlusion rat model. Chinese Medical Journal
2000;113:836-9.

12. Shukla V, Coumoul X, Wang RH, et al. RNA interference and inhibition of MEK-ERK signaling prevent abnormal skeletal phenotypes in a mouse model of craniosynostosis. Nat Genet 2007;39:1145-50.

13. Hsieh CP, Chiou YL, Lin CY. Hyperbaric oxygenstimulated proliferation and growth of osteoblasts may be mediated through the FGF-2/MEK/ERK 1/2/ $\mathrm{NF}-\kappa \mathrm{B}$ and $\mathrm{PKC/JNK}$ pathways. Connect Tissue Res 2010;51:497-509.

14. Yang $\mathrm{Y}$, Wei H, Zhou X, et al. Hyperbaric oxygen promotes neural stem cell proliferation by activating vascular endothelial growth factor/extracellular signalregulated kinase signaling after traumatic brain injury. Neuroreport 2017;28:1232-8.

15. Chen T, Zhou Q, Gao Y, et al. Rapid establishment of mild cognitive impairment models in rats. Chinese Journal of Experimental Surgery 2018;35:958-61.

16. Cui X, Zuo P, Zhang Q, et al. Chronic systemic D-galactose exposure induces memory loss, neurodegeneration, and oxidative damage in mice: protective effects of R-alpha-lipoic acid. J Neurosci Res 2006;84:647-54.

17. Zhang Q, Li X, Cui X, et al. D-galactose injured neurogenesis in the hippocampus of adult mice. Neurol Res 2005;27:552-6.

18. Chen X, Li Y, Chen W, et al. Protective Effect of Hyperbaric Oxygen on Cognitive Impairment Induced by D-Galactose in Mice. Neurochem Res 2016;41:3032-41.

19. Zhao B, Pan Y, Wang Z, et al. The effect of hyperbaric oxygen treatment on hippocampal neurons apoptotic and expression of p38 mitogen activated protein kinases in Alzheimer disease rat model. International Journal of Anesthesiology and Resuscitation 2015;36:226-30.

20. Alsina FC, Ledda F, Paratcha G. New insights into the control of neurotrophic growth factor receptor signaling: implications for nervous system development and repair. J Neurochem 2012;123:652-61.

21. Irving EA, Barone FC, Reith AD, et al. Differential activation of MAPK/ERK and p38/SAPK in neurones and glia following focal cerebral ischaemia in the rat. Brain Res Mol Brain Res 2000;77:65-75.

22. Hicks SD, Parmele KT, Defranco DB, et al. Hypothermia differentially increases extracellular signal-regulated kinase and stress-activated protein kinase/c-jun terminal kinase activation in the hippocampus during reperfusion after asphyxial cardiac 
arrest. Neuroscience 2000;98:677-85.

23. Liu J, Li R, Cui J, et al. Regulating effects of U0126 on the extracellular signal regulated kinase pathway after subarachnoid hemorrhage in mice. Chinese Journal of
Neuroanatomy 2012;28:283-8.

(English Language Editor: J. Gray)

Cite this article as: Lin Y, Lin X, Zheng X, Liu F, Ye C, Huang L, Zhou Q, Chen T, Lin L. Hyperbaric oxygen therapy cognitive function in a rat model of mild cognitive impairment via ERK signaling. Ann Palliat Med 2020;9(5):3472-3480. doi: 10.21037/apm-20-1716 